\title{
Analysis of 120 Pectoralis Major Flaps for Head and Neck Reconstruction
}

\author{
Young Sun You ${ }^{1}$, Chul Hoon Chung ${ }^{1}$, Yong Joon Chang ${ }^{1}$, Kuyl Hee Kim ${ }^{1}$, Sung Won Jung ${ }^{1}$, \\ Young Soo Rho ${ }^{2}$ \\ Departments of ${ }^{1}$ Plastic and Reconstructive Surgery, ${ }^{2}$ Otolaryngology-Head and Neck Surgery, Hallym University Medical Center, Seoul, \\ Korea
}

Background A pectoralis major flap is one of the standard tools for the reconstruction of defects of the head and neck. Despite the technical advancement in free tissue transfer in head and neck reconstruction, the benefits of a pectoralis major flap should not be overlooked. The purpose of this study is to evaluate our 17 years of experience in reconstructing defects of the head and neck region using the pectoralis major flap.

Methods We retrospectively reviewed the medical records of 112 patients (120 cases) who underwent pectoralis major flap operations for head and neck reconstruction during a period ranging from 1994 to 2010.

Results In our series, no total necrosis of the flap occurred. Of the total cases, 30.8\% presented with flap-related complications. Major complications occurred in 20\% of all of the cases but were then all successfully treated. The male sex was correlated with the occurrence of overall complications $(P=0.020)$ and major complications $(P=0.007)$. Preoperative albumin levels of $<3.8 \mathrm{~g} / \mathrm{dL}$ were correlated with the formation of fistula $(P=0.030)$. Defects of the hypopharynx were correlated with the occurrence of major complications $(P=0.019)$ and the formation of fistula $(P=0.012)$. Secondary reconstructions were correlated with the occurrence of overall complications $(P=0.013)$ and the formation of fistula $(P=0.030)$.

Conclusions A pectoralis major flap is still considered to be a safe, versatile one-stage reconstruction procedure in the management of the defects of head and neck and the protection of the carotid artery.

Keywords Pedicled flap / Complications / Risk factors
Correspondence: Chul Hoon Chung Department of Plastic and Reconstructive Surgery, Hallym University Medical Center, 150 Seongan-ro, Gangdong-gu, Seoul 134-701, Korea

Tel: $+82-2-2224-2246$

Fax: +82-2-489-0010

E-mail: c21ps@hanmail.net

This article was presented at the 68th Congress of the Korean Society of Plastic and Reconstructive Surgeons on Novermber 4-7, 2010 in Seoul, Korea.

No potential conflict of interest relevant to this article was reported.

Received: 30 May 2012 • Revised: 18 Jul 2012 • Accepted: 1 Aug 2012

pISSN: 2234-6163 • elSSN: 2234-6171 • http://dx.doi.org/10.5999/aps.2012.39.5.522• Arch Plast Surg 2012;39:522-527

\section{INTRODUCTION}

In cases of tissue defects of the head and neck where vital anatomical structures are present, it is mandatory to consider not only functional factors such as respiration, mastication, swallowing, and phonation but also esthetic factors. With the recent advancement in the microsurgery techniques, the free flap has become a primary choice in head and neck reconstruction. This is because, in a variety of recipient sites, both functional and esthetic impairments can be minimized with the use of tissues whose characteristics are similar to those of the defect sites. Currently, the free flap is routinely preferred by surgeons. Nevertheless, a pectoralis major flap is also indicated for patients with a poor general condition, those for whom a complete resection 
of the tumor cannot be performed, those for whom a free flap is not indicated but are in need of a salvage operation because of a failure of a previous free flap, those with local problems such as fistula, and those who are in need of the coverage of the exposed vessels for prevention and treatment purposes. Since it was first reported by Ariyan [1] as an island pedicle flap in 1979, the pectoralis major flap has been the standard one that is commonly used for head and neck reconstruction not only because it is stable but also because it can be elevated both promptly and easily.

Given the above background, we conducted this study to appreciate the significance of the pectoralis major flap in head and neck reconstruction, for which we evaluated the indications of this technique, the types of defect sites, the size of flap, postoperative complications, and their related risk factors.

\section{METHODS}

We retrospectively reviewed the medical records of 112 patients who underwent pectoralis major flap surgery for head and neck reconstruction in the Department of Plastic and Reconstructive Surgery of our medical institution during a period ranging from 1994 to 2010. In our series, there were a total of 120 cases of pectoralis major flap surgery and these include eight cases in which a contralateral pectoralis major flap was performed as a revision surgery because of complications such as fistula, carotid artery rupture, and a partial necrosis of the pre-existing pectoralis major flap.

We classified the indications for a pectoralis major flap into the following causes of use: the primary site of malignant tumor, postoperative complications, carotid artery protection, pharyngostoma closure, and an incomplete resection of the tumor (Table 1). We also classified cases of tumor metastasis according to the primary sites.

To evaluate the treatment outcomes, we analyzed the flaprelated complications during the hospital stay, defined as the length of time that elapsed until patients were discharged since they underwent surgery. To do this, we classified the flap-related complications into major complications requiring an operation and minor complications for which conservative treatment could be performed. If more than one complication was involved, the final one was chosen.

In our series, risk factors of complications include sex, age $\geq 71$-year-old, a degree of obesity defined as body mass index of $>25 \mathrm{~kg} / \mathrm{m}^{2}$, preoperative albumin levels of $<3.8 \mathrm{~g} / \mathrm{dL}$, a past history of radiotherapy, the sites of reconstruction, systemic diseases (diabetes mellitus, hypertension, and cardiovascular diseases) and their correlations with the complications.
Table 1. Summary of use of the pectoralis major flap

\begin{tabular}{|c|c|}
\hline Indication & No. of cases \\
\hline \multicolumn{2}{|l|}{ Malignancy } \\
\hline \multicolumn{2}{|l|}{ Oral cavity } \\
\hline Mobile tongue & 8 \\
\hline Floor of mouth & 1 \\
\hline Floor of mouth with mandible & 1 \\
\hline Buccal & 1 \\
\hline Hard palate & 1 \\
\hline \multicolumn{2}{|l|}{ Oropharynx } \\
\hline Pharyngeal wall & 5 \\
\hline Base of tongue & 4 \\
\hline Soft palate & 1 \\
\hline Retromolar trigone & 1 \\
\hline Hypopharynx & 14 \\
\hline \multicolumn{2}{|l|}{ Overlying skin invasion } \\
\hline Parotid gland & 4 \\
\hline Submandibular gland & 1 \\
\hline Thyroid gland & 4 \\
\hline Lymph nodes & 12 \\
\hline \multicolumn{2}{|l|}{ Postoperative complications } \\
\hline \multicolumn{2}{|l|}{ Fistula } \\
\hline Oral cavity & 6 \\
\hline Oropharynx & 3 \\
\hline Hypopharynx & 16 \\
\hline Necrosis of neck skin & 7 \\
\hline \multicolumn{2}{|l|}{ Previous flap necrosis } \\
\hline Oral cavity & 3 \\
\hline Oropharynx & 1 \\
\hline Neck & 1 \\
\hline \multicolumn{2}{|l|}{ Wound dehiscence } \\
\hline Oral cavity & 2 \\
\hline Pharyngostoma closure & 3 \\
\hline Carotid artery rupture & 2 \\
\hline Protection of carotid artery & 15 \\
\hline \multicolumn{2}{|l|}{ Incomplete tumor resection } \\
\hline Hypopharynx & 1 \\
\hline Neck & 2 \\
\hline Total & 120 \\
\hline
\end{tabular}

In addition, we also classified our clinical series of patients into two groups: the group where a pectoralis major flap was used for primary reconstruction and that where it was used for secondary reconstruction for the treatment of complications due to previous treatments. Then, we compared the incidence of complications between the two groups.

Statistical analysis was performed using SPSS ver. 19.0 (SPSS Inc., Chicago, IL, USA). P-values for each variable were calculated using Fisher's exact probability test. Statistical significance was accepted as $\mathrm{P}<0.05$.

\section{RESULTS}

The current study enrolled a total of 112 patients $(n=112)$, who comprised 90 men and 22 women. The mean age of the patients 
was 61.0 years (range, 34 to 86 years). Of the 90 men who underwent pectoralis major flap surgery, 8 patients underwent the operation twice, corresponding to a total of 98 cases. All of the 22 women only underwent the procedure once.

In Table 1, pectoralis major flaps are classified according to the cause of use. There were 37 cases for which a pectoralis major flap was used following the resection of malignant tumors; these include 12 cases of oral cancer, 11 cases of oropharyngeal cancer, and 14 cases of hypopharyngeal cancer based on the sites of primary occurrence.

Of the patients with hypopharyngeal cancer, there was one who underwent an incomplete resection of the tumor. This case was classified as an incomplete tumor resection.

There were 21 patients who had skin metastases. Reconstruction should therefore be performed for these patients, who comprised 4 cases of parotid gland cancer, 1 case of submandibular gland cancer, 4 cases of thyroid gland cancer, and 12 cases of metastasis to the cervical lymph node. All of the 50 cases of oral cancer, oropharyngeal cancer, hypopharyngeal cancer, and metastasis to the cervical lymph node proved to be squamous cell carcinomas. There were a total of 4 cases of parotid gland cancer and these included 1 case of mucoepidermoid carcinoma, 1 case of malignant pleomorphic adenoma, 1 case of acinic cell carcinoma, and 1 case of sarcoma. One case of submandibular gland cancer proved to be mucoepidermoid carcinoma. There were 4 cases of thyroid cancer, all of which proved to be papillary carcinomas.

There were a total of 44 cases of postoperative complications for which a pectoralis major flap needed to be performed due to complications of a previous operation. These included 25 cases of fistula, seven cases of skin necrosis on the neck, five cases of necrosis of the pre-existing flap, 2 cases of carotid artery rupture, 3 cases of pharyngostoma closure and 2 cases of wound dehiscence in the oral cavity. Additionally, there were 15 cases of carotid artery protection and 3 cases of an incomplete resection of the tumor due to extensive metastases to multiple tissues.

By the types of pectoralis major flap, there were 107 cases of myocutaneous flap, 12 cases of muscle flap, and 1 case of pectoralis major osteomyocutaneous flap involving the fifth rib. There were also cases of muscle flap; these included 7 cases of carotid artery protection, 2 cases of tumor resection due to an extensive presence of skin metastasis on the neck draining into the cervical lymph node, 2 cases of an extensive skin involvement due to metastasis of the cervical lymph nodes, and 1 case of an incomplete resection of cancer on the neck. A pectoralis major osteomyocutaneous flap was used to reconstruct the mandible and mouth floor where squamous cell carcinomas had invaded.

There were 8 patients for whom a pectoralis major flap surgery was performed twice. There were 4 patients in whom a contralateral pectoralis major musculocutaneous flap was inevitable due to the formation of fistula following the resection of a tumor using an ipsilateral pectoralis major musculocutaneous flap. There were 2 patients for whom a contralateral pectoralis major musculocutaneous flap was performed again due to the partial necrosis of a free flap following the resection of a tumor using an ipsilateral pectoralis major musculocutaneous flap and free flap. There was one patient for whom a pectoralis major musculocutaneous flap was used twice to protect the carotid artery because both carotid arteries were sequentially ruptured postoperatively. In another patient, following a resection of the tumor and ipsilateral pectoralis major musculocutaneous flap, a contralateral pectoralis major musculocutaneous flap was also used due to tumor metastasis to the cervical lymph node because of recurrence.

The size of the skin paddle of the pectoralis major musculocutaneous flap ranged from $2 \times 3 \mathrm{~cm}$ to $10 \times 15 \mathrm{~cm}$. The size of the skin paddle was the largest in cases in which the circumferential defect of the hypopharynx was reconstructed. It was $8 \times 12 \mathrm{~cm}$ in cases in which a direct closure of the recipient site was performed.

In a total of 76 cases, a pectoralis major flap was used for primary reconstruction. These included 58 cases of primary malignant tumor, 15 cases of carotid artery protection, and 3 cases of an incomplete resection of the tumor. In addition, a pectoralis major flap was also used for secondary reconstruction in a total of 44 cases. These included 39 cases of postoperative complications, 3 cases of pharyngostoma closure, and 2 cases of carotid artery protection that had been performed due to postoperative rupture of the carotid artery.

There were 40 cases of primary tumor with no past history of treatment, and there were 19 salvage cases in which the tumor recurred although they received treatment.

In 37 cases (30.8\%), a pectoralis major flap was used for reconstruction to shorten the operation time because of poor general conditions due to systemic diseases. In addition, it was also used for the following reasons: external skin defect (28 cases, $23.3 \%$ ), postoperative complication (35 cases, $29.2 \%$ ), carotid artery protection (17 cases, $14.2 \%$ ), and an incomplete resection of the tumor (3 cases, $2.5 \%$ ) (Table 2). With regard to poor general conditions, there were 12 patients who had both severe diabetes mellitus and hypertension, 8 with cachexia, 8 with ischemic heart disease, 5 with renal failure, and 4 patients with liver cirrhosis.

There were 37 cases (30.8\%) of flap-related complications; these included 8 cases of partial necrosis, 12 cases of fistula, 10 cases of wound dehiscence, 6 cases of infection, and 1 case of 
Table 2. Reasons for choosing the pectoralis major flap

\begin{tabular}{|lc|}
\hline Reason & No. of cases \\
\hline Poor general condition & 37 \\
Skin coverage & \\
Cheek & 4 \\
$\quad$ Neck & 24 \\
Postoperative complication & \\
Fistula & 25 \\
Previous flap necrosis & 5 \\
Wound dehiscence & 2 \\
Pharyngostoma closure & 3 \\
Protection of carotid artery & 17 \\
Incomplete tumor resection & 3 \\
Total & 120 \\
\hline
\end{tabular}

bleeding. Furthermore, there were 24 cases of major complications, and these included 4 cases of partial necrosis, 12 cases of fistula, 4 cases of wound dehiscence, 3 cases of infection, and 1 case of bleeding. There were 13 cases of minor complications and these included 4 cases of partial necrosis, 6 cases of wound dehiscence, and 3 cases of infection. The complications occurred in 35 men and 2 women. All of the 24 cases of major complications occurred in men. In addition, minor complications occurred in 11 men and 2 women.

For the treatment of partial necrosis, one of the flap-related complications, the following measures were taken: a trapezius musculocutaneous flap ( 1 case), a split-thickness skin graft (1 case), and a direct closure ( 1 case). For the treatment of fistula, the following measures were taken: a palatal mucoperiosteal flap (1 case), a contralateral pectoralis major musculocutaneous flap ( 4 cases), a radial forearm free flap ( 1 case), a latissimus dorsi musculocutaneous flap ( 1 case), pharyngostoma formation ( 3 cases), and a direct closure ( 2 cases). Direct closure was performed in all of the 4 patients with wound dehiscence and a partial-thickness skin graft was performed in all of the 3 patients with infection. There was 1 case of bleeding in the pectoralis major muscle, which was controlled by suture ligation (Table 3 ).

At the donor sites, the complications included 1 case of skin necrosis, 1 case of infection, and 3 cases of bleeding. In our series, there were 13 patients who died of sepsis or cardiovascular failure.

Statistical analysis was performed to identify the correlations between the flap-related complications and their risk factors. This showed that the complications were not correlated with such risk factors as age, obesity, a past history of radiotherapy, salvage operation, systemic diseases, primary reconstruction, or the reconstruction of the oral cavity.

The incidence of overall complications $(\mathrm{P}=0.020)$ and major complications $(\mathrm{P}=0.007)$ was significantly higher in men than
Table 3. Treatment for pectoralis major flap-related major complications

\begin{tabular}{|c|c|c|c|}
\hline Complication & Site & Treatment & $\begin{array}{l}\text { No. of } \\
\text { cases }\end{array}$ \\
\hline \multicolumn{4}{|l|}{ Partial necrosis } \\
\hline & Buccal & Trapezius MC flap & 1 \\
\hline & Hypopharynx & Direct closure & 2 \\
\hline & Neck & STSG & 1 \\
\hline \multicolumn{4}{|l|}{ Fistula } \\
\hline & Hard palate & $\begin{array}{c}\text { Palatal mucoperiosteal } \\
\text { flap }\end{array}$ & 1 \\
\hline & Floor of mouth & Pectoralis major MC flap & 2 \\
\hline & Base of tongue & Pharyngostoma & 2 \\
\hline & Hypopharynx & Radial forearm free flap & 1 \\
\hline & & Pharyngostoma & 1 \\
\hline & & Pectoralis major MC flap & 2 \\
\hline & & Latissimus dorsi MC flap & 1 \\
\hline & & Direct closure & 2 \\
\hline \multicolumn{4}{|l|}{ Wound dehiscence } \\
\hline & Tonsil & Direct closure & 2 \\
\hline & $\begin{array}{l}\text { Retromolar } \\
\text { trigone }\end{array}$ & Direct closure & 1 \\
\hline & Neck & Direct closure & 1 \\
\hline \multicolumn{4}{|l|}{ Infection } \\
\hline & Neck & STSG & 3 \\
\hline \multicolumn{4}{|l|}{ Bleeding } \\
\hline & $\begin{array}{l}\text { Pectoralis } \\
\text { major muscle }\end{array}$ & Suture ligation & 1 \\
\hline Total & & & 24 \\
\hline
\end{tabular}

in women. Low preoperative albumin levels $(<3.8 \mathrm{~g} / \mathrm{dL})$ were significantly correlated with the incidence of fistula formation $(\mathrm{P}=0.030)$. The frequency of hypopharyngeal reconstruction was significantly correlated with the incidence of major complications $(\mathrm{P}=0.019)$ and fistula formation $(\mathrm{P}=0.012)$. Finally, the frequency of secondary reconstruction was significantly correlated with the incidence of overall complications $(\mathrm{P}=0.013)$ and fistula formation $(\mathrm{P}=0.030)$ (Table 4).

\section{DISCUSSION}

In head and neck reconstruction using a free flap, various flap designs are available depending on the characteristics of the defect site. In addition, their functional outcomes have been reported to be excellent. The authors have therefore used a free flap for reconstruction in the majority of primary cases [2-4]. However, if the operation time should be shortened because of poor general conditions due to systemic diseases or if a free flap is unavailable because of vascular disorders such as atherosclerosis, a pedicled flap can also be used for the reconstruction. Koh et al. [5] measured the operation time of the reconstruction using a pectoralis major flap and a free flap in patients with de- 
Table 4. Correlation between potential risk factors and subcategories of complications

\begin{tabular}{|c|c|c|c|c|c|c|c|c|c|c|c|c|}
\hline \multirow{2}{*}{$\begin{array}{l}\text { Factor Cx. } \\
\text { (cases) }\end{array}$} & \multicolumn{3}{|c|}{ Male } & \multicolumn{3}{|c|}{ Albumin $<3.8 \mathrm{~g} / \mathrm{dL}$} & \multicolumn{3}{|c|}{ Hypopharynx } & \multicolumn{3}{|c|}{ Secondary reconstruction } \\
\hline & $Y(98)$ & $N(22)$ & P-value ${ }^{\text {a) }}$ & $Y(52)$ & $N(68)$ & P-value & $Y(31)$ & $N(89)$ & P-value & $Y(44)$ & $N(76)$ & P-value \\
\hline Overall Cx. (37) & 35 & 2 & $0.020^{b)}$ & 18 & 19 & 0.550 & 14 & 23 & 0.069 & 20 & 17 & $0.013^{b)}$ \\
\hline Major Cx. (24) & 24 & 0 & $0.007^{b)}$ & 10 & 14 & 1.000 & 11 & 13 & $0.019^{b)}$ & 13 & 11 & 0.059 \\
\hline Minor Cx. (13) & 11 & 2 & 1.000 & 8 & 5 & 0.236 & 3 & 10 & 1.000 & 7 & 6 & 0.225 \\
\hline Partial necrosis (8) & 8 & 0 & 0.348 & 3 & 5 & 1.000 & 3 & 5 & 0.424 & 4 & 4 & 0.463 \\
\hline Fistula (12) & 12 & 0 & 0.120 & 9 & 3 & $0.030^{b)}$ & 7 & 5 & $0.012^{b)}$ & 8 & 4 & $0.030^{b)}$ \\
\hline Dehiscence (10) & 8 & 2 & 1.000 & 3 & 7 & 0.511 & 1 & 9 & 0.450 & 5 & 5 & 0.495 \\
\hline Infection (6) & 6 & 0 & 0.591 & 3 & 3 & 1.000 & 2 & 4 & 0.648 & 3 & 3 & 0.668 \\
\hline Bleeding (1) & 1 & 0 & 1.000 & 0 & 1 & 1.000 & 1 & 0 & 0.258 & 0 & 1 & 1.000 \\
\hline
\end{tabular}

fects of the oral cavity and oropharynx and then compared the time for each of the two flaps. According to that study, the mean operation time was $76 \pm 7$ minutes (range, 54 to 105 minutes) in cases of a pectoralis major flap reconstruction and $145 \pm 11$ minutes (range, 115 to 205 minutes) in those of a free flap reconstruction.

We have chosen a pectoralis major flap for the reconstruction of head and neck defects for the following reasons: poor general conditions $(30.8 \%)$, the treatment of postoperative complications $(29.2 \%)$, skin coverage (23.3\%), carotid artery protection (14.2\%), and an incomplete resection of the tumor (2.5\%). It is apparent that a free flap can also be used for the treatment of postoperative complications as performed in one patient who underwent resection of hypopharyngeal cancer and for whom the reconstruction was performed using a pectoralis major musculocutaneous flap. In this patient, however, because approximately $2 / 3$ of the skin paddle of the pectoralis major musculocutaneous flap was necrotized, the pectoralis major musculocutaneous flap was removed and the reconstruction was performed using a radial forearm free flap.

In our series, all of the pectoralis major flaps were harvested with conventional techniques, but they were not true island flaps. We included the muscle with a width of $3 \mathrm{~cm}$ in making a pedicle to avoid the excessive traction of the thoracoacromial vessel for the purpose of increasing the stability of the flap. Notwithstanding the transposition of the flap via a supraclavicular route, there were no cases in which the shortness of the pedicle had caused a problem. The wound closure without tension could also be achieved because of sufficient pedicle length.

The skin paddle was positioned on the medial side in women in order to reduce the amount of breast tissue in the flap. A pectoralis major flap was used because a relatively smaller skin paddle was needed to treat postoperative complications such as fistula, wound dehiscence, partial necrosis of the previous flap, or pharyngostoma. In cases in which the skin paddle of the pectoralis major musculocutaneous flap was relatively small, the flap was elevated including the subcutaneous fat layer around the skin paddle at a width of $2 \mathrm{~cm}$ while the skin paddle was being attached to the flap to improve the vascularization. Presumably, this technical aspect of flap dissection might have reduced complications and thus brought about better outcomes.

Flap-related complications occurred in $30.8 \%$ of all of the patients; these complications included partial necrosis (6.7\%), fistula formation (10\%), wound dehiscence (8.3\%), infection (5\%), and bleeding (0.8\%). According to previously published studies, the incidence of overall complications ranged between $35.5 \%$ and $63 \%$. These studies have also noted that the incidence range of total necrosis, partial necrosis, fistula, wound dehiscence, infection, and bleeding were $0-2.7 \%$, 4-29\%, 5.5-29\%, $2.8-26 \%, 2-24 \%$, and $1-7 \%$, respectively [6-14]. Our clinical series included no cases of total necrosis of the flap and a relatively low incidence of complications. Flap-related complications, such as infection, wound dehiscence, necrosis, and fistula, can also lead to other complications. Therefore, these complications were classified for the final status just before the treatment of complications. In our series, there were 13 patients who died of sepsis or cardiovascular disease, but none of our clinical series of patients died of flap-related complications.

With regard to the correlation between flap-related complications and risk factors, Shah et al. [7] noted that flap necrosis was correlated with an age of $>70$ years, being female, obesity, preoperative albumin levels $(<4.0 \mathrm{~g} / \mathrm{dL})$, the reconstruction of the oral cavity, and systemic diseases. Corten et al. [13] and McLean et al. [15] also reported that there was a significant correlation between a past history of radiotherapy and overall complications. In addition, Pinto et al. [14] reported that there was a significant correlation between hypopharyngeal reconstruction and overall complications. Furthermore, Chepeha et al. [4] also reported that there was a significant correlation between hypopharyngeal reconstruction and major complications. According 
to Corten et al. [13], however, there was a correlation between partial flap necrosis and the reconstruction of the oral cavity and oropharynx and a correlation between fistula formation and secondary reconstruction.

We also analyzed the correlation between flap-related complications and risk factors. This showed that the overall incidence of complications was significantly higher in men. In addition, it was also relatively high in patients who underwent secondary operation for the treatment of complications. The incidence of major complications was significantly higher in men and correlated with hypopharyngeal reconstruction. The incidence of fistula formation was significantly correlated with lower preoperative albumin levels, hypopharyngeal reconstruction, and secondary reconstruction. However, such variables as age, being female, a past history of radiotherapy, systemic diseases, and the reconstruction of the oral cavity were not significant risk factors.

Shah et al. [7] reported that the incidence of flap-related complications was relatively high in obese patients with larger-sized breasts. Our results showed, however, that all the major complications occurred in men. Presumably, this might be not only because the majority of our clinical series of patients were rather thin but also because a pectoralis major musculocutaneous flap was elevated on the medial side of the breast to leave the breast tissue intact in forming the skin pedicle. In patients whose preoperative albumin levels were relatively low, it is necessary to preoperatively supplement with a sufficient amount of albumin. In addition, it is also necessary to elevate the flap including the subcutaneous fat layer with the subcutaneous fat layer attached to the tissue adjacent to the skin pedicle. This is essential for improving the vascularity of the skin pedicle, especially in cases of the hypopharyngeal reconstruction. This should also be accompanied by a more delicate suture.

A pectoralis major flap is advantageous in that it can be used for reconstruction by a one-time operation. In addition, it is stable because of a definitely reliable vascularity and also has a shorter operation time.

It can therefore be concluded that a pectoralis major flap could be used alternatively to a free flap in such special cases as poor general conditions due to a systemic disease, external skin coverage, or carotid artery protection.

\section{REFERENCES}

1. Ariyan S. The pectoralis major myocutaneous flap. A versatile flap for reconstruction in the head and neck. Plast Reconstr Surg 1979;63:73-81.

2. Urken ML, Weinberg H, Buchbinder D, et al. Microvascular free flaps in head and neck reconstruction. Report of 200 cases and review of complications. Arch Otolaryngol Head Neck Surg 1994;120:633-40.

3. Tsue TT, Desyatnikova SS, Deleyiannis FW, et al. Comparison of cost and function in reconstruction of the posterior oral cavity and oropharynx. Free vs pedicled soft tissue transfer. Arch Otolaryngol Head Neck Surg 1997; 123:731-7.

4. Chepeha DB, Annich G, Pynnonen MA, et al. Pectoralis major myocutaneous flap vs revascularized free tissue transfer: complications, gastrostomy tube dependence, and hospitalization. Arch Otolaryngol Head Neck Surg 2004;130:181-6.

5. Koh KS, Eom JS, Kirk I, et al. Pectoralis major musculocutaneous flap in oropharyngeal reconstruction: revisited. Plast Reconstr Surg 2006;118:1145-9.

6. Baek SM, Lawson W, Biller HF. An analysis of 133 pectoralis major myocutaneous flaps. Plast Reconstr Surg 1982;69: 460-9.

7. Shah JP, Haribhakti V, Loree TR, et al. Complications of the pectoralis major myocutaneous flap in head and neck reconstruction. Am J Surg 1990;160:352-5.

8. Kroll SS, Goepfert H, Jones M, et al. Analysis of complications in 168 pectoralis major myocutaneous flaps used for head and neck reconstruction. Ann Plast Surg 1990;25:93-7.

9. Mehta S, Sarkar S, Kavarana N, et al. Complications of the pectoralis major myocutaneous flap in the oral cavity: a prospective evaluation of 220 cases. Plast Reconstr Surg 1996; 98:31-7.

10. IJsselstein CB, Hovius SE, ten Have BL, et al. Is the pectoralis myocutaneous flap in intraoral and oropharyngeal reconstruction outdated? Am J Surg 1996;172:259-62.

11. Vartanian JG, Carvalho AL, Carvalho SM, et al. Pectoralis major and other myofascial/myocutaneous flaps in head and neck cancer reconstruction: experience with 437 cases at a single institution. Head Neck 2004;26:1018-23.

12. Milenovic A, Virag M, Uglesic V, et al. The pectoralis major flap in head and neck reconstruction: first 500 patients. J Craniomaxillofac Surg 2006;34:340-3.

13. Corten EM, Schellekens PP, Hage JJ, et al. Clinical outcome after pedicled segmental pectoralis major island flaps for head and neck reconstruction. Ann Plast Surg 2009;63:292-6.

14. Pinto FR, Malena CR, Vanni CM, et al. Pectoralis major myocutaneous flaps for head and neck reconstruction: factors influencing occurrences of complications and the final outcome. Sao Paulo Med J 2010;128:336-41.

15. McLean JN, Carlson GW, Losken A. The pectoralis major myocutaneous flap revisited: a reliable technique for head and neck reconstruction. Ann Plast Surg 2010;64:570-3. 